\title{
Kinetics of bilirubin and liver enzymes is useful for predicting of liver graft-versus-host disease
}

\author{
M. KREJCI ${ }^{1, *}$, J. KAMELANDER ${ }^{1}$, Z. POSPISIL ${ }^{2}$, J. MAYER ${ }^{1}$ \\ ${ }^{1}$ Department of Internal Medicine, Hematology and Oncology, University Hospital Brno and Masaryk University, Jihlavska 20,62500 Brno, \\ Czech Republic; ${ }^{2}$ Masaryk University, Department of Mathematics and Statistics, Janackovo nam. 2a, 60200 Brno, Czech Republic
}

${ }^{*}$ Correspondence: $m k r e j c i @ f n b r n o . c z$

Received October 3, 2011 / Accepted November 11, 2011

\begin{abstract}
Graft-versus-host disease (GVHD) is the most frequent complication after allogeneic hematopoietic cell transplantation. We analyzed the kinetics of bilirubin and liver enzymes in 47 cases with liver GVHD and in 47 cases without GVHD after allogeneic transplantation for various hematological malignancies.

The duration of an liver GVHD episode (LGVHD) was defined as the interval from the point when the criteria of LGVHD were met to the decrease to $<2$ upper normal limit (UNL) for aminotransferases or bilirubin $<34 \mu \mathrm{mol} / \mathrm{l}$ for bilirubin. The imminent LGVHD episode was defined as the interval from the start of continuous increase ( $\geq 3$ consecutive rising values) of bilirubin and liver enzymes above UNL to the point of LGVHD diagnosis.

The number of imminent LGVHD episodes, and median length in days were as follows: bilirubin (39;5), ALT(28;12), $\operatorname{AST}(9 ; 12)$, GGTP(34;9), and ALP(13;14). Statisticallly significant associations between asymptomatic continuous increase of bilirubin, ALT, and GGTP and later liver GVHD manifestation were found ( $\mathrm{p}=0.004, \mathrm{p}=0.008, \mathrm{p}=0.005$, respectively). The asymptomatic continuous increase in bilirubin, ALT, and GGTP occurred at a median of 5, 12, and 9 days before liver GVHD episode, respectively.

In the control group without GVHD, median levels of bilirubin and liver enzymes were within normal limits and no continuous increase was observed.

Kinetics of bilirubin and liver enzymes is useful for predicting of liver GVHD. A continuous increase of bilirubin and/or ALT, GGTP before the standard liver GVHD criteria are met can be a sign of coming liver GVHD.
\end{abstract}

Key words: liver GVHD, kinetics, bilirubin, liver enzymes

Graft-versus-host disease (GVHD) is the most frequent complication after allogeneic hematopoietic cell transplantation [1-3]. Acute GVHD is usually reported in stages or grades based on the extent of organ involvement; any organ can be affected alone or in combination [4,5]. For liver GVHD, the classical parameter is bilirubin.

The current classification of liver GVHD is based on a pivotal paper by Glucksberg et al [6]. In this article, the authors mention not only the elevation of bilirubin, but also raised levels of serum glutamic oxaloacetic acid transaminase. However, alterations in the level of transaminases and/or other liver enzymes have not become a part of the current classification systems which only use bilirubin [4,7]. Liver enzyme measurements are an important part of the diagnosis and monitoring of the hepatitic variant of liver GVHD $[8,9]$.
The hepatitic variant of GVHD is characterized by elevations of serum aminotransferase levels (alanine aminotransferase (ALT) or aspartate aminotransferase (AST) $>10$ of upper normal limit, UNL) [9]. However, liver GVHD has its onset well before the serum markers reach the diagnostic threshold and early changes in these parameters have not been studied in detail. Moreover, a crucial histological feature of liver GVHD is the damage to the small bile ducts [10], which may increase plasma levels of the other two easily measurable liver enzymes, alkaline phosphatase (ALP) and gamma glutamyl transpeptidase (GGTP).

Our study was aimed at the detection of early abnormalities of markers of liver GVHD, in particular on the dynamics and kinetics of liver enzymes and signs of imminent liver GVHD. 


\section{Patients and Methods}

Patients with liver GVHD. This study is a retrospective analysis of $47 \mathrm{GVHD}$ liver episodes in 26 patients (pts) (median age 40 years; range 20 -56 years) from our database of 112 pts after allogeneic transplantation (62 with GVHD, 50 without GVHD). The underlying diseases were as follows: chronic myeloid leukemia, $n=9$; acute myeloid leukemia, $n=6$; chronic lymphocytic leukemia, $\mathrm{n}=2$; myelodysplastic syndrome, $\mathrm{n}=2$; other hematological malignancies, $n=7$. The two preparative regimens most frequently used were a reduced intensity conditioning (RIC) regimen containing busulfan + fludarabine + and antithymocyte globulin (ATG Fresenius) $(n=10)$, and a myeloablative regimen that included busulfan + cyclophosphamide $(n=12)$. Peripheral blood was the source of stem cells in all cases (human leukocyte antigen (HLA) identical sibling, $\mathrm{n}=12$; matched unrelated donors (MUD), $\mathrm{n}=14$ ). GVHD prophylaxis usually consisted of the cyclosporine A and methotrexate (for myeloablative conditioning), cyclosporine alone or cyclosporine and mycophenolate mofetil (for reduced intensity conditioning). Patient characteristics are shown in Table 1. Classic liver GVHD with elevation of bilirubin $\geq 34$ $\mu \mathrm{mol} / \mathrm{l}$ was present in $77 \%$ of GVHD episodes $(36 / 47)$, elevation of ALT $>10$ UNL in $62 \%$ of GVHD episodes (29/47), and elevation of AST $>10$ UNL in $23 \%$ of GVHD cases (11/47).

Control group without GVHD. It included 26 pts with hematological malignancies after SCT without GVHD. The characteristics of the control group were similar to GVHD patients regarding age (median age 41 years; range $22-58$ years), type of preparative regimens (RIC in 12 cases, myeloablative regimens in 14 cases), type of donors (identical sibling in 13 cases, MUD in 13 pts). Kinetics of bilirubin and liver enzymes (ALT, AST, GGTP, ALP) were evaluated at 47 same time points after SCT as in patients with GVHD.

Methods. Retrospectively were identified pts with liver GVHD diagnosed on the basis of bilirubin and/or aminotransferases levels. All pts had combined forms of GVHD (involvement of liver and/or skin and/or oral mucosa and/or

Table 1. Patient characteristics

\begin{tabular}{lc}
\hline Number of patients: & 26 \\
\hline Age (years): median; range & $40 ; 20-56$ \\
Conditioning: RIC/myeloablative & $14 / 12$ \\
Number of evaluated GVHD liver episodes: & 47 \\
Elevation of bilirubin $\geq 34 \mu \mathrm{mol} / \mathrm{l}:$ & $36 \mathrm{cases}, 77 \%$ \\
Elevation of ALT (ALT $>10$ of UNL): & 29 cases, 62\% \\
Elevation of AST (AST $>10$ of UNL): & 11 cases, 23\% \\
Number of evaluated imminent GVHD liver episodes: & 47 \\
Elevation of bilirubin (range: $>$ UNL to < 34 $\mu$ mol/l): & 39 cases, 83\% \\
Elevation of ALT (range: $>$ UNL-10 of UNL): & 28 cases, 60\% \\
Elevation of AST (range: $>$ UNL-10 of UNL): & 9 cases, 19\% \\
Elevation of GGTP (> UNL) & 34 cases, 72\% \\
Elevation of ALP ( $>$ UNL) & 13 cases, 28\% \\
\hline
\end{tabular}

gut) and had histopathologic confirmation of GVHD from organ biopsy in the time of elevation of bilirubin and/or aminotransferases (liver biopsy was performed at 18 pts (69\%), other organ biopsy at 8 pts (31\%)).

Excluded were cases with other possible etiologies of liver damage or elevation of bilirubin and/or aminotransferases, including viral infections (hepatitis B and C, CMV), hemolysis, secondary hemochromatosis.

The GVHD liver episode was defined as the number of days from the time of fulfilment the standard laboratory diagnostic criteria for liver GVHD or for hepatitic liver GVHD (bilirubin $\geq 34 \mu \mathrm{mol} / \mathrm{l}$ and/or ALT $>10$ of UNL and/or AST $>10$ of UNL) $[4,9]$ to the decrease to $<2$ UNL for aminotransferases or to $<$ $34 \mu \mathrm{mol} / \mathrm{l}$ for bilirubin values. For every GVHD liver episode, we have assessed the duration of bilirubin and liver enzyme elevation in days. The increased levels of bilirubin and liver enzymes were expressed as multiples of UNL. We have attempted to detect a possible correlation between continuous asymptomatic increase of bilirubin or liver enzymes and later manifestation of liver GVHD.

For all laboratory tests (bilirubin, ALT, AST, GGTP or ALP), we have identified the day from which there was a continuous increase ( $\geq 3$ consecutive rising values) in that particular parameter, and we have determined the time interval from start of this increase (i.e. the point when the continuously increasing levels exceeded UNL) to the date of definite diagnosis of liver GVHD according to the standard criteria. We have designated this interval as imminent liver GVHD episode.

For hospitalized pts, the laboratory parameters were usually measured on a daily basis or every other day.

Statistics: Standard descriptive statistics were computed for all quantitative characteristics. Calculations for the statistical analysis were performed using the STATISTICA data analysis software system, version 9.0. Univariate analysis was performed with differences analyzed using the long-rank test. P-values $<0.050$ were considered significant.

\section{Results}

GVHD liver episodes. There were 47 liver GVHD episodes in 26 pts. The minimal, maximal, and median length of liver GVHD episodes were in days as follows: 2, 415, 24; for bilirubin only: $(0,415,10)$, for ALT: $(0,157,13)$, and for AST: $(0,54,0)$. Duration of GVHD liver episodes is shown in Table 2.

The respective median values (expressed as multiples of UNL) for bilirubin, ALT, AST, GGTP, and ALP during GVHD

Table 2. Duration of GVHD liver episodes in days

\begin{tabular}{lcccc}
\hline & Total & Bilirubin & ALT & AST \\
\hline Number of episodes & 47 & 47 & 47 & 47 \\
Minimum & 5 & 0 & 0 & 0 \\
Maximum & 151 & 151 & 117 & 54 \\
Median & 15 & 10 & 12 & 7 \\
\hline
\end{tabular}


liver episodes were as follows: 1.7, 4.4, 1.6, 8.0, and 1.1. This shows that GGTP is probably the most sensitive marker of liver damage (Table 3 and Figure 1).

Imminent liver GVHD episodes. The dynamics of bilirubin and four liver enzymes (ALT, AST, GGTP, ALP) before their levels reached the diagnostic criteria for liver GVHD were evaluated. The number of imminent liver GVHD episodes, where a continuous increase $(\geq 3$ consecutive rising values) in the laboratory parameters was seen before reaching the diagnostic liver GVHD values (e.g. bilirubin $\geq 34 \mu \mathrm{mol} / \mathrm{l}$ and/or ALT $>10$ of UNL and/or AST $>10$ of UNL) was shown in Table 1 . The minimum, maximum, and median length in days of that increase were as follows: bilirubin $(0,70,5)$, ALT $(0,71,12)$, AST $(0,72,12)$, GGTP $(0,70,9)$, and $\operatorname{ALP}(0,57$, 14 (Table 4.) In imminent LGVHD, median levels of bilirubin, ALT, AST, GGTP and ALP (in multiples of UNL) were 1.4, 3.8, $1.5,5.4$, and 1.4 , respectively.

There was a statistically significant association between asymptomatic continuous increase of bilirubin (39/47) and later manifestation of liver GVHD ( $\mathrm{p}=0.004)$.

We have also studied possible association between the continuous increase of AST (9/47) and ALP (13/47) and later development of liver GVHD but no statistically significant correlations were observed $(\mathrm{p}=0.478$ and $\mathrm{p}=0.518$, respectively).

However, there were statistically significant associations between continuous increase of ALT (28/47) and GGTP $(34 / 47)$ and later liver GVHD ( $\mathrm{p}=0.008$ and $\mathrm{p}=0.005$, respectively).

The asymptomatic continuous increase of bilirubin, ALT, and GGTP occurred at a median of 5, 12, and 9 days before liver GVHD episode, respectively. The results of the univariate analysis are summarised in Table 5.

Table 3. Descriptive statistics of bilirubin and liver enzymes during GVHD liver episodes expressed as multiples of upper normal limits

\begin{tabular}{lccccc}
\hline & Bilirubin & ALT & AST & GGTP & ALP \\
\hline Number of episodes & 47 & 47 & 47 & 47 & 47 \\
Minimum & 0.3 & 0.5 & 0.5 & 1.0 & 0.4 \\
Maximum & 10.8 & 15.5 & 4.5 & 41.4 & 3.5 \\
Median & 1.7 & 4.4 & 1.6 & 8.0 & 1.1 \\
\hline
\end{tabular}

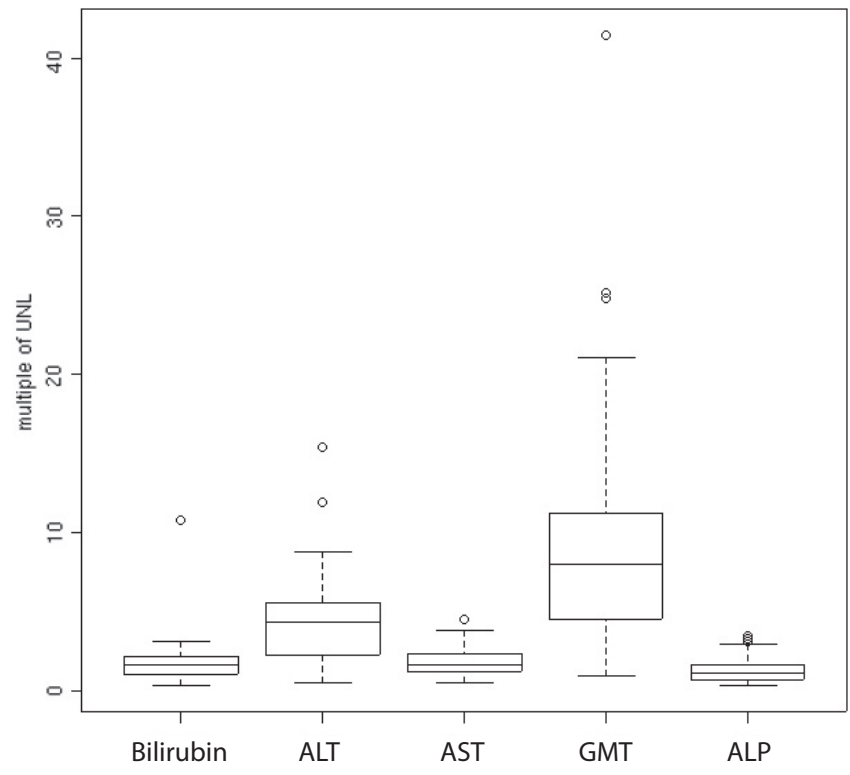

Figure 1. Kinetics of bilirubin and liver enzymes during liver GVHD episode: multiples of upper normal limits

Control group without GVHD. Dynamics of bilirubin and liver enzymes were evaluated in 26 pts at 47 time points that were identical to those used in pts with GVHD. Asymptomatic elevations of bilirubin, ALT, AST, GGTP and ALP were present in $15 \%, 26 \%, 22 \%, 29 \%$ and $32 \%$ of the control group patients, respectively. None of the control group patients had a continuous increase of bilirubin and liver enzymes. Median levels of bilirubin and liver enzymes were within the normal limits in the control group. Elevations of bilirubin, ALT, AST,

Table 4. Duration of bilirubin and liver enzyme elevation (in days) in the imminent liver GVHD

\begin{tabular}{lccccc}
\hline & Bilirubin & ALT & AST & GGTP & ALP \\
\hline Number of episodes & 47 & 47 & 47 & 47 & 47 \\
Minimum & 0 & 0 & 0 & 0 & 0 \\
Maximum & 70 & 71 & 72 & 70 & 57 \\
Median & 5 & 12 & 12 & 9 & 14 \\
\hline
\end{tabular}

Table 5. Associations between asymptomatic continuous increase of bilirubin and liver enzymes and later manifestation of liver GVHD: results of univariate analysis

\begin{tabular}{lcccc}
\hline & $\begin{array}{c}\text { Number of imminent GVHD episodes with continuous } \\
\text { increase }(\geq 3 \text { consecutive rising values })\end{array}$ & $\begin{array}{c}\text { Later manifestation of } \\
\text { liver GVHD }\end{array}$ & $\begin{array}{c}\text { Hazard ratio } \\
(95 \% \text { CI })\end{array}$ & P-value \\
\hline Bilirubin & 39 & 47 & $1.6(1.07-1.92)$ & 0.004 \\
ALT & 28 & 47 & $1.3(1.04-1.63)$ & 0.008 \\
AST & 9 & 47 & $0.75(0.51-1.01)$ & 0.478 \\
GGTP & 34 & 47 & $1.4(1.05-1.82)$ & 0.005 \\
ALP & 13 & 47 & $0.79(0.49-1.12)$ & 0.518 \\
\hline
\end{tabular}


GGT and ALP were mild and intermittent with the following median levels (in multiples of UNL): 1.2, 1.4, 1.3, 1.6, and 1.3, respectively.

\section{Discussion}

According to several studies, GVHD is considered a serious post-transplant or post-donor lymphocyte infusion complication $[8,9,11,12]$ and is one of the major causes of allogeneic hematopoietic stem cell transplant-related morbidity and mortality. Elevation of liver enzymes in patients with clinical or histological evidence of cutaneous or intestinal GVHD is usually attributable to liver GVHD [13]. Liver biopsy is a useful method for the confirmation of liver GVHD [9], but it carries a significant risk and the rate of serious complications with the use of transjugular liver biopsy has ranged from 1.3 to $20.2 \%$ [14].

Elevation of bilirubin has been established as a standard parameter for acute liver GVHD and is used in current GVHD classification systems [4,7]. In clinical differentiation of icterus (bilirubin $\geq 40-50 \mu \mathrm{mol} / \mathrm{l}$ ) it is necessary to take various types of icterus into consideration. A detection of unconjugated and conjugated bilirubin in icterus etiology is important factor for icterus differentiation (praemicrosomial type with predominance of unconjugated bilirubin, microsomial type with similar levels of unconjugated and conjugated bilirubin and postmicrosomial icterus with predominance of conjugated bilirubin). It is important that in GVHD only total bilirubin will be used generally. In our cohort of patients, we evaluated only overall bilirubin, but detection of conjugated and unconjugated bilirubin can be interesting method for exact type of hyperbilirubinaemia in GVHD patients.

In differential diagnosis of icterus after allogeneic transplant, veno-occlusive disease of liver (VOD) will be excluded. Veno-occlusive disease was diagnosed in the basis of Jones criteria [15]: hyperbilirubinaemia with bilirubin $\geq 34 \mu \mathrm{mol} / \mathrm{l}$ and any two of following symptoms: ascites, painful hepatomegaly and unexplained weight gain $\geq 5 \%$ from baseline within 20 days of allogeneic transplant. In our cohort of patients, no patient with VOD was observed.

The occurrence of liver enzyme abnormalities during the course of liver GVHD is probably underestimated, and these parameters are considered a part of diagnostic criteria for GVHD. However, the usefulness of liver enzyme measurement for the diagnosis of liver GVHD has been emphasized by some authors [16-18]. The serum ALP level was described as a sensitive diagnostic marker for liver GVHD with a parallel rise in serum bilirubin level [16]. Serum levels of ALT and AST are frequently elevated, especially during the early stages of GVHD [17]. Hepatitic variant of liver GVHD with elevations of ALT and AST [9] or elevations of ALT, AST and ALP has been described previously [18].

Measurement of all liver tests (bilirubin, ALT, AST, ALP, GGTP) to characterize the dynamics of liver GVHD has been carried out by few research groups [19]. Chronic liver GVHD usually presents as a progressive cholestasis with elevations of bilirubin, ALP and GGTP along with other organ involvement [20], but chronic liver GVHD presenting as an acute hepatitis has been observed, too [8].

According to our findings, a combination of bilirubin and/or liver enzyme elevations was observed in all cases of liver GVHD episodes. In some cases of liver GVHD, hyperbilirubinemia dominates, whereas in other episodes, extreme elevation of liver enzymes is a key clinical feature with bilirubin in the normal range or only marginally elevated.

Statisticallly significant associations between asymptomatic continuous increase of bilirubin, ALT, and GGTP and later liver GVHD manifestation were found in our study. The asymptomatic continuous increase in bilirubin, ALT, and GGTP occurred at a median of 5, 12, and 9 days before liver GVHD episode, respectively.

The rigorous and regular testing of all routine liver enzymes is useful for early identification of cases with imminent liver GVHD before the classical GVHD criteria are fulfilled.

Kinetics of bilirubin and liver enzymes is useful for predicting of liver GVHD. A continuous increase of bilirubin, ALT and/or GGTP before the standard liver GVHD criteria are met can be a sign of imminent liver GVHD. Prospective studies are needed to confirm and extend our results.

Acknowledgements: This work was supported in part by The Czech Leukemia Study Group for Life, CELL, and the Research Grant MSM 0021622430.

\section{References}

[1] MARTIN PJ, CARPENTER PA, SANDERS JE, FLOWERS ME. Diagnosis and clinical management of chronic graft-versushost disease. In J Hematol 2004; 79: 221-228.

[2] GOKER H, HAZNEDAROGLU IC, CHAO NJ. Acute graft-vs-host disease: pathobiology and management. Exp Hematol 2001; 29: 259-277. http://dx.doi.org/10.1016/S0301$\underline{472 X(00) 00677-9}$

[3] DEEG HJ. How I treat refractory acute GVHD. Blood 2007; 109: 4119-4126. http://dx.doi.org/10.1182/blood-2006-12041889

[4] PRZEPIORKA D, WEISDORF D, MARTIN P, KLINGEMANN HG, BEATTY P et al. Consensus conference on acute GVHD grading. Bone Marrow Transplant 1995; 15: 825-828.

[5] CAHN JY, KLEIN JP, LEE SJ, MILPIED N, BLAISE D et al. Prospective evaluation of 2 acute graft-versus-host (GVHD) grading systems: a joint Société Française de Greffe de Moëlle et Thérapie Cellulaire (SFGM-TC), Dana Farber Cancer Institute (DFCI), and International Bone Marrow Transplant Registry (IBMTR) prospective study. Blood 2005;106: 1495500. http://dx.doi.org/10.1182/blood-2004-11-4557

[6] GLUCKSBERG H, STORB R, FEFER A, BUCKNER CD, NEIMAN PE et al. Clinical manifestations of graft-versus-host disease in human recipients of marrow from HLA matched sibling donors. Transplantation 1974; 18: 295-304. http:// dx.doi.org/10.1097/00007890-197410000-00001 
[7] ROWLINGS PA, PRZEPIORKA D, KLEIN JP, GALE RP, PASSWEG JR et al. IBMTR Severity Index for grading acute graft-versus-host disease: retrospective comparison with Glucksberg grade. Br J Haematol 1997; 97: 855-864. http:// dx.doi.org/10.1046/j.1365-2141.1997.1112925.x

[8] STRASSER SI, SHULMAN HM, FLOWERS ME, REDDY R, MARGOLIS DA et al. Chronic graft-versus-host disease of the liver: presentation as an acute hepatitis. Hepatology 2000; 32: 1265-1271. http://dx.doi.org/10.1053/jhep.2000.20067

[9] AKPEK G, BOITNOTT JK, LEE LA, HALLICK JP, TORBENSON M et al. Hepatitic variant of graft-versus-host disease after donor lymphocyte infusion. Blood 2002; 100: 3903-3907. http://dx.doi.org/10.1182/blood-2002-03-0857

[10] MAENG H, LEE JH, CHEONG JW, LEE ST, HAHN JS et al. Chronic graft-versus-host diesease of the liver presenting as an acute hepatitis following nonmyeloablative hematopoietic stem cell transplantation. Int J Hematol 2004; 79: 501-504. http://dx.doi.org/10.1532/IJH97.A10319

[11] BACIGALUPO A. Management of acute graft-versushost disease. Br J Hematol 2007; 137: 87-98. http://dx.doi. org/10.1111/j.1365-2141.2007.06533.x

[12] COURIEL D, HUMBERTO C, CHAMPLIN R, KOMANDURI K. Acute graft-versus-host disease: pathophysiology, clinical manifestations and management. Cancer 2004; 101: 19361946. http://dx.doi.org/10.1002/cncr.20613

[13] MCDONALD GB, SHULMAN HM, SULLIVAN KM, SPENCER GD. Intestinal and hepatic complications of human bone marrow transplantation. Gastroenterology 1986; 90: 770-784.

[14] MA SY, AU WY, LIE AK, NG IO, LEUNG AY et al. Liver graft-versus-host disease after donor lymphocyte infusion for relapses of hematologic malignancies post allogeneic hematopoietic stem cell transplantation. Bone Marrow Transplant 2004; 34: 57-61. http://dx.doi.org/10.1038/sj.bmt.1704522

[15] JONES RJ, LEE KS, BESCHORNER WE, VOGEL VE et al. Venooclusive disease of the liver following bone marrow transplantation. Transplantation 1987; 44: 778-783. http://dx.doi. org/10.1097/00007890-198712000-00011

[16] YASMINEH WG, FILIPOVICH AH, KILLEEN AA. Serum 5 'nucleotidase and alkaline phoshatase-highly predictive liver function tests for the diagnosis of graft-versus-host disease in bone marrow transplant recipients. Transplantation 1989; 48: 809-814. http://dx.doi.org/10.1097/00007890-198911000$\underline{00017}$

[17] STRASSER SI, MCDONALD GB. Gastrointestinal and hepatic complications. In: Thomas ED, Blumde KG, Foman SJ (eds). Hematopoietic Cell Transplantation. Blackwell Science: Boston, MA, 1999, pp 627-658.

[18] MA SY, AU WY, NG IO, LIE AK, LEUNG AY et al. Hepatitic graft-versus-host disease after hematopoietic stem cell transplantation: clinicopathologic features and prognostic implication. Transplantation 2004; 77: 1252-1259. http://dx.doi. org/10.1097/01.TP.0000120383.30088.A4

[19] FRIED RH, MURAKAMI CS, FISHER LD, WILLSON RA, SULLIVAN KM et al. Ursodeoxycholic acid treatment of refractory chronic graft-versus-host disease of the liver. Ann Intern Med 1992; 116: 624-629.

[20] SULLIVAN KM, SHULMAN HM, STORB R, WEIDEN PL, WITHERSPOON RP et al. Chronic graft-versus-host disease in 52 patients: adverse natural course and successful treatment with combination immunosuppression. Blood 1981; 57: 267276. 